\title{
A propensity score-matched case-control study of laparoscopy and laparotomy for endometrial cancer
}

\author{
Licerio Miguel ${ }^{1}$ (D), Julio Cesar Rosa e Silva ${ }^{1}$ (D), Omero Benedito Poli Neto ${ }^{1}$ (D), \\ Daniel Guimarães Tiezzi ${ }^{1} \mathbb{B}$, Jurandyr Moreira de Andrade ${ }^{1} \mathbb{B}$, \\ Francisco Jose Candido dos Reis ${ }^{1 *}$
}

\section{SUMMARY}

OBJECTIVE: A surgery is essential for the management of early endometrial carcinoma. Due to the comorbidities associated with the disease, the complications of surgery are common. Laparoscopic surgery may reduce surgical complications but also have oncological risks. We aimed to compare recurrence and overall survival (OS) associated with laparoscopy and laparotomy for early endometrial cancer. METHODS: We included women treated for presumed early endometrial carcinoma at the Clinics Hospital of Ribeirão Preto Medical School from January 1998 to December 2017. We designed a 1:2 propensity score-matched case-control and compared the patients' characteristics, short-term outcomes, recurrence, and OS.

RESULTS: A total of 252 women were included in this study, 168 underwent laparotomy, and 84 underwent laparoscopy. The two groups were well balanced according to most of the variables, and obesity was a characteristic of patients in both groups. Laparoscopy was associated with increased surgical time (194.7 min vesus $165.6 \mathrm{~min} ; \mathrm{p}<0.001)$ and reduced rate of surgical complications $(6.5 \%$ versus $0 ; p=0.038$ ). Laparoscopic surgery was not associated with the risk of tumor recurrence (HR: $0.41,95 \% \mathrm{Cl} 0.14-1.19, p=0.100)$ or all-cause mortality (HR: $0.49,95 \% \mathrm{Cl} 0.18-1.35, \mathrm{p}=0.170)$.

CONCLUSION: Laparoscopy was safe in terms of oncological outcomes and was associated with a lower rate of surgical complications. Our data support the use of minimally invasive surgery as the preferential approach in the management of early endometrial carcinoma. KEYWORDS: Endometrial carcinoma. Laparoscopy. Laparotomy. Survival.

\section{INTRODUCTION}

Uterine corpus cancer accounts for $7 \%$ of all female malignant tumors and $4 \%$ of estimated cancer deaths ${ }^{1}$. Among the uterine corpus tumors, endometrial carcinoma corresponds to $97 \%{ }^{2}$. Endometrial carcinoma is associated with the well-defined risk factors such as obesity, nulliparity, or genetic risk ${ }^{3}$. Compared with other female tumors, endometrial carcinoma has a good prognosis. Its mortality-to-incidence ratio is 0.24 , which is lower than breast cancer $(0.32)$, ovarian cancer $(0.63)$, or cervical cancer $(0.55)^{4}$. However, endometrial carcinoma frequently coexists with obesity ${ }^{5}$ that in association with comorbidities such as hypertension, diabetes, and pulmonary disease may lead to incomplete staging, increased surgical time, increased blood loss, and abdominal wall infection and dehiscence ${ }^{6,7}$.

Over the past two decades, the use of laparoscopy and, more recently, robotic surgery for the treatment of endometrial

\footnotetext{
'Universidade de São Paulo, Faculdade de Medicina de Ribeirão Preto, Departamento de Ginecologia e Obstetrícia - Ribeirão Preto (SP), Brazil. *Corresponding author: fjcreis@usp.br

Conflicts of interest: the authors declare there is no conflicts of interest. Funding: this study was financially supported in part by the Coordenação de Aperfeiçoamento de Pessoal de Nível Superior (CAPES), Brazil, Finance Code 001, and in part by the Fundação de Apoio ao Ensino, Pesquisa e Assistência do Hospital das Clínicas da Faculdade de Medicina de Ribeirão Preto da Universidade de São Paulo (FAEPA) (Grant number 1856/2018). F. J. Candido dos Reis (Grant number 303210/2018-4) was funded by the Conselho Nacional de Desenvolvimento Científico e Tecnológico (CNPq). Received on February 15, 2021. Accepted on March 14, 2021.
} 
cancer has expanded substantially. The minimally invasive techniques reduce complications related to the surgery ${ }^{8}$. Several studies showed the advantages of the laparoscopic technique over conventional laparotomy. The main short-term advantages relate to pain reduction and better postoperative quality of life ${ }^{9-11}$. Some risks, such as urinary tract or intestinal injury and tumor dissemination, may also be associated with the minimally invasive techniques. Current evidence, from clinical trials and meta-analysis, points to the equivalence between conventional laparotomy and laparoscopic surgery in terms of the long-term outcomes ${ }^{12,13}$.

The data from the clinical trials are the gold standard for evaluating interventions in oncology. However, the real-world clinical data are essential to characterize the advantages and risks of interventions in clinical practice. This study aimed to compare laparoscopy with laparotomy, concerning the overall survival (OS) and the disease-free survival (DFS) using a propensity score-matched case-control from a single institution.

\section{METHODS}

\section{Study design}

We designed a 1:2 propensity score-matched case-control of early endometrial carcinoma treated at Clinics Hospital of Ribeirão Preto Medical School from the year 1998 to 2017. The Institutional Board for Ethics in Research approved the study (CAAE: 81115817.5.0000.5440), and the informed consent was waived. The study was reported following the strengthening the reporting of observational studies in epidemiology (STROBE) checklist for the case-control studies.

\section{Inclusion and exclusion criteria}

Patients with a histological diagnosis of endometrial cancer without clinical or image evidence of cervical or extrauterine disease were eligible. The exclusion criteria were as follows: advanced disease at diagnosis, previous surgical treatment for endometrial cancer, surgery not performed for other reasons (e.g., impaired performance status), and prior chemotherapy or radiotherapy for the endometrial cancer treatment.

\section{Surgical procedures}

During this period, deciding whether to use laparoscopic surgery or traditional laparotomy followed the surgeon's preference. For laparoscopic surgeries, we used umbilical Veress needle entry in all cases, $\mathrm{CO}_{2}$ for abdominal distention, and the Trendelenburg position. The bipolar electrical devices were used for vessel sealing. Vaginal approaches were used for colpotomy and section of cardinal/uterosacral ligaments.
Midline incisions were used as the preferred routes for laparotomies. In both types of surgeries, the decision on when to perform lymphadenectomy was based on preoperative image and biopsy (i.e., histology and grade) and intraoperative findings (i.e., the estimation of myometrial invasion). As a general rule, women with grade I/II superficial endometrioid tumors did not undergo lymphadenectomy.

Variables and statistics

The variables collected were as follows: type of surgery, age at diagnosis, body mass index (BMI), sonographic uterine volume, tumor histology, the International Federation of Gynecology and Obstetrics (FIGO) stage of tumor, tumor grade, preoperative CA-125 levels, adjuvant treatment (i.e., radiotherapy and chemotherapy), duration of anesthesia, duration of surgery, lymphadenectomy and number of nodes, intraoperative complications, surgical postoperative complications, clinical postoperative complications, thromboembolic events, blood transfusion needed, hospital postoperative stay, and 5-year DFS and 5-year OS.

The disease-free survival (DFS) is defined as the interval from the time of primary surgery to any documented disease recurrence. The OS is defined as the interval from primary surgery to death by any cause. For patients who were still alive at the time of analysis, DFS and OS were censored at the last follow-up date.

A total of 400 cases (i.e., 84 laparoscopic surgeries and 316 open surgeries) were eligible for the study. In this group, we had the randomly missing data for CA-125, BMI, and uterine volume. Therefore, the values were imputed ${ }^{14}$ using the Amelia $\mathrm{R}$ package. After the imputation of the missing data, we calculated the propensity score using the variables, such as age, race, education, BMI, previous surgery, histology, stage, grade, uterine weight, CA-125, and preoperative comorbidities. Then, 84 laparoscopic cases were matched 1:2 to open surgery cases, resulting in 252 cases analyzed.

Summary statistics were used to compare the variables between laparoscopy and laparotomy groups. Qualitative variables are displayed using frequencies and percentages. The Kaplan-Meier product-limit estimator and the Cox proportional-hazards regression model were used for the survival analysis. The data manipulation and statistical analysis were performed with $\mathrm{R}$ version 4.0.2.

\section{RESULTS}

In our matched case-control study, 168 patients underwent conventional laparotomy and 84 underwent laparoscopic surgery. The characteristics of the included patients and outcomes are presented in Table 1. The two groups were well balanced 
Table 1. Characteristics of patients and outcomes according to the type of surgery.

\begin{tabular}{|c|c|c|c|}
\hline Variable & Laparoscopy & Laparotomy & $p$-value \\
\hline Cases & $n=84$ & $n=168$ & \\
\hline Age (years) & $64.2(11.6)$ & $64.5(10.1)$ & 0.856 \\
\hline Body mass index $\left(\mathrm{kg} / \mathrm{m}^{2}\right)$ & $33.7(8.7)$ & $32.9(7.2)$ & 0.492 \\
\hline Uterine volume $\left(\mathrm{cm}^{3}\right)$ & $105.8(73.5)$ & $109.3(87.6)$ & 0.759 \\
\hline \multicolumn{4}{|l|}{ Histologic type } \\
\hline Endometrioid carcinoma & $78(92.9 \%)$ & $155(92.3 \%)$ & 0.964 \\
\hline Carcinosarcoma & $3(3.6 \%)$ & $7(4.2 \%)$ & \\
\hline Serous carcinoma & $2(2.4 \%)$ & $3(1.8 \%)$ & \\
\hline Mixed cell carcinoma & $1(1.2 \%)$ & $3(1.8 \%)$ & \\
\hline \multicolumn{4}{|l|}{ FIGO stage } \\
\hline IA & $39(46.4 \%)$ & $76(45.2 \%)$ & 0.917 \\
\hline IB & $27(32.1 \%)$ & $57(33.9 \%)$ & \\
\hline II & $9(10.7 \%)$ & $19(11.3 \%)$ & \\
\hline IIIA & $5(6 \%)$ & $7(4.2 \%)$ & \\
\hline IIIB & $2(2.4 \%)$ & $2(1.2 \%)$ & \\
\hline IIIC & $2(2.4 \%)$ & $7(4.2 \%)$ & \\
\hline \multicolumn{4}{|l|}{ Grade } \\
\hline 1 & $42(50 \%)$ & $78(46.4 \%)$ & 0.864 \\
\hline$\|$ & $32(38.1 \%)$ & $68(40.5 \%)$ & \\
\hline III & $10(11.9 \%)$ & $22(13.1 \%)$ & \\
\hline CA-125 (U/mL) & $44.7(73.4)$ & $32.0(100.4)$ & 0.304 \\
\hline Duration of anesthesia (min) & $233.5(58.5)$ & $204.6(60.1)$ & $<0.001$ \\
\hline Duration of surgery (min) & $194.7(55.7)$ & $165.6(58.2)$ & $<0.001$ \\
\hline \multicolumn{4}{|l|}{ Lymphadenectomy } \\
\hline Pelvic & $64(76.2 \%)$ & $111(66.1 \%)$ & 0.19 \\
\hline Pelvic and aortic & $0(0 \%)$ & $2(1.2 \%)$ & \\
\hline None & $20(23.8 \%)$ & $55(32.7 \%)$ & \\
\hline Number of nodes & $7.0(8.8)$ & $8.0(6.0)$ & 0.809 \\
\hline Intraoperative complication & $3(3.6 \%)$ & $7(4.2 \%)$ & 1 \\
\hline Surgical postoperative complication & $0(0 \%)$ & $11(6.5 \%)$ & 0.038 \\
\hline Clinical postoperative complication & $5(6 \%)$ & $7(4.2 \%)$ & 0.754 \\
\hline Thromboembolic event & $1(1.2 \%)$ & $3(1.8 \%)$ & 1.000 \\
\hline Blood transfusion & $1(1.2 \%)$ & $13(7.7 \%)$ & 0.065 \\
\hline Postoperative hospital stay (days) & $2.0(0.0)$ & $2.0(1.0)$ & 0.336 \\
\hline \multicolumn{4}{|l|}{ Radiotherapy } \\
\hline None & $53(63.1 \%)$ & $100(59.5 \%)$ & 0.503 \\
\hline External & $9(10.7 \%)$ & $13(7.7 \%)$ & \\
\hline Brachytherapy & $3(3.6 \%)$ & $13(7.7 \%)$ & \\
\hline Combined & $19(22.6 \%)$ & $42(25 \%)$ & \\
\hline Adjuvant chemotherapy & $5(6 \%)$ & $11(6.5 \%)$ & 1.000 \\
\hline
\end{tabular}

FIGO: International Federation of Gynecology and Obstetrics 
according to most of the variables. Obesity was an important characteristic of both groups. Endometrioid histology (92.9 and $92.3 \%)$, stage 1 (78.5 and 79.1\%), and low grade (88.1 and $86.9 \%$ ) were predominant in laparoscopic and open surgery groups, respectively. Adjuvant radiotherapy was used for $36.9 \%$ of laparoscopic and $40.5 \%$ of open surgery cases. Adjuvant chemotherapy was used for $6 \%$ of laparoscopic and $6.5 \%$ of open surgery cases. Both groups presented similar results on the percentage of women submitted to lymphadenectomy, the number of nodes harvested, intraoperative complications, postoperative clinical complications, thromboembolic events, blood transfusion, and postoperative hospital stay. We observed increased anesthesia time (233.5 min versus $204.6 \mathrm{~min} ; \mathrm{p}<0.001)$ and surgery time $(194.7 \mathrm{~min}$ versus
$165.6 \mathrm{~min} ; \mathrm{p}<0.001)$ for laparoscopy. Laparoscopy was associated with a lower rate of surgical complications than laparotomy $(6.5 \%$ versus $0 ; \mathrm{p}=0.038)$.

Table 2 shows the hazard ratio for several prognostic variables associated with recurrence and all-cause mortality. Laparoscopic surgery was not associated with neither the risk of tumor recurrence (HR: $0.41,95 \% \mathrm{CI} 0.14-1.19 ; \mathrm{p}=0.100$ ) nor the all-cause mortality (HR: 0.49, 95\%CI 0.18-1.35; $\mathrm{p}=0.170$ ). We observed an increased risk of recurrence associated with serous histology, tumor stage, and tumor grade. The risk of the all-cause mortality increased with age at diagnosis, serous histology, and tumor stage.

The Kaplan-Meier curves showed no significant differences in DFS or OS or comparing the two groups (Figure 1).

Table 2. Cox regression model for recurrence and all-cause mortality.

\begin{tabular}{|c|c|c|c|c|c|c|}
\hline & \multicolumn{3}{|c|}{ Recurrence } & \multicolumn{3}{|c|}{ All-cause mortality } \\
\hline Variable & $\mathrm{HR}$ & $95 \% \mathrm{Cl}$ & $\mathrm{p}$ & $\mathrm{HR}$ & $95 \% \mathrm{Cl}$ & $\mathrm{p}$ \\
\hline \multicolumn{7}{|l|}{ Surgery type } \\
\hline Laparotomy & Ref & & & & & \\
\hline Laparoscopy & 0.41 & $(0.14-1.19)$ & 0.100 & 0.49 & $(0.18-1.35)$ & 0.170 \\
\hline Age & 1.02 & $(0.97-1.06)$ & 0.477 & 1.06 & $(1.01-1.1)$ & 0.015 \\
\hline \multicolumn{7}{|l|}{ Histology } \\
\hline Endometrioid & Ref & & & Ref & & \\
\hline Serous & 23.63 & $(1.38-405.52)$ & 0.029 & 9.28 & $(1.54-56.07)$ & 0.015 \\
\hline Carcinosarcoma & 1.67 & $(0.3-9.22)$ & 0.557 & 2.64 & $(0.42-16.4)$ & 0.298 \\
\hline \multicolumn{7}{|l|}{ FIGO stage } \\
\hline IA & Ref & & & Ref & & \\
\hline IB & 2.72 & $(0.74-9.96)$ & 0.132 & 3.53 & $(0.98-12.75)$ & 0.054 \\
\hline$\|$ & 6.1 & $(1.25-29.68)$ & 0.025 & 3.66 & $(0.65-20.55)$ & 0.140 \\
\hline IIIA & 35.78 & $(5.12-249.94)$ & $<0.001$ & 5.59 & $(0.72-43.46)$ & 0.099 \\
\hline IIIB & 61.68 & $(6.55-580.91)$ & $<0.001$ & 61.01 & $(6.67-558.06)$ & $<0.001$ \\
\hline IIIC & 75.7 & (7.78-736.48) & $<0.001$ & 37.4 & $(4.48-312.5)$ & $<0.001$ \\
\hline \multicolumn{7}{|l|}{ Grade } \\
\hline I & Ref & & & Ref & & \\
\hline$\|$ & 4.59 & $(1.22-17.2)$ & 0.024 & 0.99 & $(0.36-2.76)$ & 0.990 \\
\hline III & 13.85 & $(2.39-80.12)$ & 0.003 & 1.29 & $(0.27-6.23)$ & 0.755 \\
\hline \multicolumn{7}{|l|}{ Radiotherapy } \\
\hline None & Ref & & & Ref & & \\
\hline External & 1.41 & $(0.28-7.03)$ & 0.679 & 0.2 & $(0.02-1.71)$ & 0.141 \\
\hline Brachytherapy & 0.18 & (0.03-1.09) & 0.062 & 0 & (0-Inf) & 0.997 \\
\hline Combined & 0.4 & $(0.12-1.33)$ & 0.134 & 0.77 & $(0.25-2.42)$ & 0.655 \\
\hline Chemotherapy & 0.06 & $(0-0.79)$ & 0.033 & 0.5 & $(0.07-3.72)$ & 0.496 \\
\hline
\end{tabular}




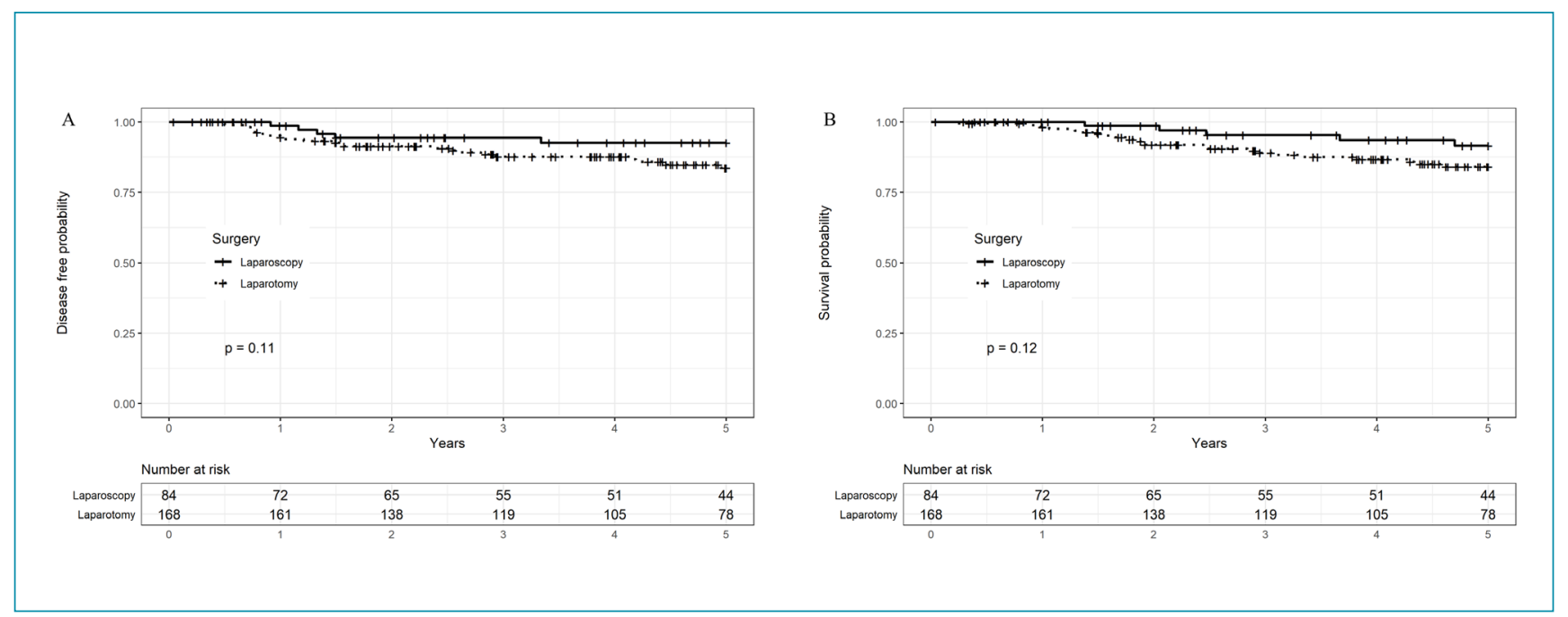

Figure 1. Kaplan-Meier curves of women with early endometrial cancer undergone laparoscopic surgery or conventional laparotomy. (A) 5-years disease-free survival. (B) 5-years overall survival.

\section{DISCUSSION}

Our results confirm that the laparoscopic surgery is safe in terms of oncological outcomes for early endometrial carcinoma. Although there was an increase of $30 \mathrm{~min}$ on the time of surgery and anesthesia, the risk of surgical complications was significantly reduced. The laparoscopic surgery was not associated with the 5-year risk of tumor relapse or all-cause mortality.

The main advantages of this study were as follows: a well-documented cohort, the comprehensive follow-up, and the concomitance over time of laparoscopy and laparotomy. The follow-up allowed the long-term observation of outcomes, and the concomitance of procedures was essential to minimize the bias due trends in survival associated with improvements in treatment quality ${ }^{15}$. Our study design also allowed comparing both the procedures' effectiveness in a real-world setting ${ }^{16}$. A limitation of our study was that laparoscopy or laparotomy was performed according to the surgeon's preference, and therefore the procedures were conducted by different surgeons who might have different skills and experience.

Compared with laparotomy, laparoscopy was associated with fewer complications. Our results are concordant with others in demonstrating a reduced risk of surgical complications in patients who underwent laparoscopic surgery, as well as the association of laparoscopy with a significantly longer operative time $e^{17,18}$. However, the clinical trials did not find differences in the surgical complications between laparotomy and laparoscopy ${ }^{19}$. A meta-analysis of the data from eight randomized controlled trials found similar intraoperative complication rates in both surgical approaches, less blood loss in laparoscopy, shorter operative time in laparotomy, and significant advantage of laparoscopy over laparotomy in terms of postoperative complications ${ }^{20}$.
Similar to others ${ }^{8}$, we found that the surgical technique was the most important variable associated with the complication rate.

There are few studies on the long-term outcomes of laparoscopic surgery published. We did not find evidence of any increment of risk of recurrence associated with laparoscopic surgery. This result is similar to a previous meta-analysis ${ }^{21}$. The GOG-LAP2 trial also confirmed the laparoscopic safety in terms of recurrence and survival $^{22}$ with a median of 5 years of follow-up. As early endometrial cancer has a high probability of cure with surgical treatment, the main causes of death among these patients are not endometrial cancer itself. The leading cause of death is the cardiovascular disease $\mathrm{e}^{23,24}$. The laparoscopic surgery is associated with fewer surgically related complications and better postoperative quality of life $\mathrm{e}^{10,25}$, which may be associated with improved general health conditions posttreatment and has the potential to reduce the all-cause mortality.

\section{CONCLUSIONS}

In conclusion, our data add to the concept that the minimally invasive technology should be applied whenever possible in these cases.

\section{AUTHORS' CONTRIBUTIONS}

LM: Data curation, Formal analysis, Investigation, Writing - review \& editing. JCRS: Validation, Writing - review $\&$ editing. OBPN: Data curation, Validation, Writing review \& editing. DGT: Data curation, Validation, Writing - review \& editing. JMA: Validation, Writing - review \& editing. FJCR: Conceptualization, Formal analysis, Funding acquisition, Methodology, Supervision, Writing - original draft. 


\section{REFERENCES}

1. Siegel RL, Miller KD, Jemal A. Cancer statistics, 2019. CA Cancer J Clin. 2019;69(1):7-34. https://doi.org/10.3322/ caac. 21551

2. Rose PG. Endometrial carcinoma. N Engl J Med. 1996;335(9):640-9. https://doi.org/10.1056/NEJM199608293350907

3. Morice P, Leary A, Creutzberg C, Abu-Rustum N, Darai E. Endometrial cancer. Lancet. 2016;387(10023):1094-108. https://doi.org/10.1016/S0140-6736(15)00130-0

4. Ferlay J, Soerjomataram I, Dikshit R, Eser S, Mathers C, Rebelo $M$, et al. Cancer incidence and mortality worldwide: sources, methods and major patterns in GLOBOCAN 2012. Int J Cancer. 2015;136(5):E359-86. https://doi.org/10.1002/ijc.29210

5. Bergström A, Pisani P, Tenet $V$, Wolk A, Adami HO. Overweight as an avoidable cause of cancer in Europe. Int J Cancer. 2001;91(3):421-30. https://doi.org/10.1002/10970215(200002)9999:9999<::aid-ijc1053>3.0.co;2-t

6. Everett E, Tamimi H, Greer B, Swisher E, Paley P, Mandel L, et al. The effect of body mass index on clinical/pathologic features, surgical morbidity, and outcome in patients with endometrial cancer. Gynecol Oncol. 2003;90(1):150-7. https:// doi.org/10.1016/s0090-8258(03)00232-4

7. Foley K, Lee RB. Surgical complications of obese patients with endometrial carcinoma. Gynecol Oncol. 1990;39(2):171-4. https://doi.org/10.1016/0090-8258(90)90427-m

8. Tozzi R, Malur S, Koehler C, Schneider A. Laparoscopy versus laparotomy in endometrial cancer: first analysis of survival of a randomized prospective study. J Minim Invasive Gynecol. 2005;12(2):130-6. https://doi.org/10.1016/j.jmig.2005.01.021

9. Janda M, Gebski V, Brand A, Hogg R, Jobling TW, Land R, et al. Quality of life after total laparoscopic hysterectomy versus total abdominal hysterectomy for stage I endometrial cancer (LACE): a randomised trial. Lancet Oncol. 2010;11(8):772-80. https://doi.org/10.1016/\$1470-2045(10)70145-5

10. Kornblith $A B$, Huang $H Q$, Walker JL, Spirtos NM, Rotmensch J, Cella D. Quality of life of patients with endometrial cancer undergoing laparoscopic international federation of gynecology and obstetrics staging compared with laparotomy: a Gynecologic Oncology Group study. J Clin Oncol. 2009;27(32):5337-42. https://doi.org/10.1200/JCO.2009.22.3529

11. Obermair A, Manolitsas TP, Leung Y, Hammond IG, McCartney AJ. Total laparoscopic hysterectomy for endometrial cancer: patterns of recurrence and survival. Gynecol Oncol. 2004;92(3):789-93. https://doi.org/10.1016/j.ygyno.2003.12.001

12. Galaal K, Bryant A, Fisher AD, Al-Khaduri M, Kew F, Lopes $A D$. Laparoscopy versus laparotomy for the management of early stage endometrial cancer. Cochrane Database Syst Rev. 2012;(9):CD006655. https://doi.org/10.1002/14651858. CD006655.pub2

13. Janda M, Gebski V, Davies LC, Forder P, Brand A, Hogg $R$, et al. Effect of total laparoscopic hysterectomy vs total abdominal hysterectomy on disease-free survival among women with stage I endometrial cancer: a randomized clinical trial. JAMA. 2017;317(12):1224-33. https://doi.org/10.1001/ jama.2017.2068
14. White IR, Royston P, Wood AM. Multiple imputation using chained equations: issues and guidance for practice. Stat Med. 2011;30(4):377-99. https://doi.org/10.1002/sim.4067

15. Inoue S, Hosono S, Ito $\mathrm{H}$, Oze I, Nishino $\mathrm{Y}$, Hattori $\mathrm{M}$, et al. Improvement in 5-year relative survival in cancer of the corpus uteri from 1993-2000 to 2001-2006 in Japan. J Epidemiol. 2018;28(2):75-80. https://doi.org/10.2188/jea.JE20170008

16. Kim HS, Lee S, Kim JH. Real-world evidence versus randomized controlled trial: clinical research based on electronic medical records. J Korean Med Sci. 2018;33(34):e213. https://doi. org/10.3346/jkms.2018.33.e213

17. Walker JL, Piedmonte MR, Spirtos NM, Eisenkop SM, Schlaerth JB, Mannel RS, et al. Laparoscopy compared with laparotomy for comprehensive surgical staging of uterine cancer: gynecologic oncology group study LAP2. J Clin Oncol. 2009;27(32):5331-6. https://doi.org/10.1200/JCO.2009.22.3248

18. Wright JD, Neugut Al, Wilde ET, Buono DL, Tsai WY, Hershman DL. Use and benefits of laparoscopic hysterectomy for stage I endometrial cancer among medicare beneficiaries. J Oncol Pract. 2012;8(5):e89-99. https://doi.org/10.1200/JOP.2011.000484

19. Mourits MJE, Bijen CB, Arts HJ, ter Brugge HG, van der Sijde $R$, Paulsen L, et al. Safety of laparoscopy versus laparotomy in early-stage endometrial cancer: a randomised trial. Lancet Oncol. 2010;11(8):763-71. https://doi.org/10.1016/S1470-2045(10)70143-1

20. Zullo F, Falbo A, Palomba S. Safety of laparoscopy vs laparotomy in the surgical staging of endometrial cancer: a systematic review and metaanalysis of randomized controlled trials. Am J Obstet Gynecol. 2012;207(2):94-100. https://doi.org/10.1016/j.ajog.2012.01.010

21. Palomba S, Falbo A, Russo T, Zullo F. Updating of a recent meta-analysis of randomized controlled trials to assess the safety and the efficacy of the laparoscopic surgery for treating early stage endometrial cancer. Gynecol Oncol. 2009;114(1):135-6. https://doi.org/10.1016/j.ygyno.2009.03.025

22. Walker JL, Piedmonte MR, Spirtos NM, Eisenkop SM, Schlaerth JB, Mannel RS, et al. Recurrence and survival after random assignment to laparoscopy versus laparotomy for comprehensive surgical staging of uterine cancer: Gynecologic Oncology Group LAP2 study. J Clin Oncol. 2012;30(7):695-700. https:// doi.org/10.1200/JCO.2011.38.8645

23. Felix AS, Bower JK, Pfeiffer RM, Raman SV, Cohn DE, Sherman ME. High cardiovascular disease mortality after endometrial cancer diagnosis: results from the surveillance, epidemiology, and end results (SEER) database. Int J Cancer. 2017;140(3):55564. https://doi.org/10.1002/ijc.30470

24. Ward KK, Shah NR, Saenz CC, McHale MT, Alvarez EA, Plaxe SC. Cardiovascular disease is the leading cause of death among endometrial cancer patients. Gynecol Oncol. 2012;126(2):176-9. https://doi.org/10.1016/j.ygyno.2012.04.013

25. Zullo F, Palomba S, Russo T, Falbo A, Costantino M, Tolino $A$, et al. A prospective randomized comparison between laparoscopic and laparotomic approaches in women with early stage endometrial cancer: a focus on the quality of life. Am J Obstet Gynecol. 2005;193(4):1344-52. https://doi. org/10.1016/j.ajog.2005.02.131 